\title{
Ambulatory Urodynamics
}

\author{
Stefan De Wachter
}

In the management of patients with SCI, a correct diagnosis and treatment of the associated neurogenic lower urinary tract dysfunction (NLUTD) is necessary to preserve renal function, prevent complications and improve patient's quality of life. Video-urodynamics (VUDS) is the gold standard test for diagnosis of NLUTD. However, the physiological nature of VUDS is often questioned because of the use of radiological contrast (which has a different density than urine) and is instilled at a lower temperature. Furthermore, the bladder is retrogradely filled, often at a rate higher than physiological filling rates. Also, the patient is placed in an artificial environment, which may also affect lower urinary tract dynamics.

Ambulatory urodynamics (AUDS) may theoretically be a more physiological test to evaluate lower urinary tract function as the bladder is filled through normal diuresis, the investigation can be performed in the patient's own environment (e.g. Home or rehab center) and can also be conducted for a longer period of time allowing the patient to perform normal activities. However, the procedure is time-consuming and technically challenging, making it more prone to artefacts.

In non-neurogenic patients AUDS has been shown to be more sensitive to detect detrusor overactivity [1], although it's currently being used when conventional UDS are inconclusive. Only limited and yet conflicting data are available on the use of AUDS in patients with neurogenic LUTD. No agreement was found in parameters relevant to the management of NLUTD between VUDS and AUDS when only one filling cycle was evaluated during AUDS [2]. On the other hand, AUDS was more sensitive to detect detrusor overactivity, an important parameter in the management of NLUTD, if an extended evaluation of nearly $5 \mathrm{~h}$ duration was used [3]. At best, based upon the available data, AUDS with extended evaluation periods may be considered if conventional VUDS is inconclusive but one should be aware that extended periods AUDS for $5 \mathrm{~h}$ may be time-consuming to perform and analyze the data and prone to artefacts.

\section{References}

1. Pannek J, Pieper P. Clinical usefulness of ambulatory urodynamics in the diagnosis and treatment of lower urinary tract dysfunction. Scand J Urol Nephrol. 2008;42:428-32.

2. Virseda-Chamorro M, Salinas-Casado J, de la Marta-Garcia M, Esteban-Fuertes M, Mendez S. Comparison of ambulatory versus video urodynamics in patients with spinal cord injury. Spinal Cord. 2014;52:551-5.

3. Martens FM, van Kuppevelt HJ, Beekman JA, Heijnen IC, D'Hauwers KW, Heesakkers JP. No primary role of ambulatory urodynamics for the management of spinal cord injury patients compared to conventional urodynamics. Neurourol Urodyn. 2010;29:1380-6.

S. De Wachter $(\bowtie)$

Department of Urology, University Hospital Antwerp,

University of Antwerp, Antwerp, Belgium

e-mail: stefan.dewachter@uantwerpen.be 